\title{
Hepatocellular Carcinoma in Korea: an Analysis of the 2015 Korean Na- tionwide Cancer Registry
}

\author{
Jun Sik Yoon ${ }^{1 *}$, Han Ah Lee ${ }^{2 *}$, Hwi Young Kim ${ }^{3}$, Dong Hyun Sinn ${ }^{4}$, Dong Ho Lee ${ }^{5}$, Suk Kyun Hong ${ }^{6}$, Ju-Yeon Cho ${ }^{7}$, Jonggi \\ $\mathrm{Choi}^{8}$, Young Chang ${ }^{9}$, Hyun-Joo Kong ${ }^{10}$, Eunyang Kim ${ }^{10}$, Young-Joo Won ${ }^{10}$, Jeong-Hoon Lee ${ }^{11}$ \\ 'Department of Internal Medicine, Busan Paik Hospital, Inje University College of Medicine, Busan; ${ }^{2}$ Department of Internal Medicine, \\ Sanggye Paik Hospital, Inje University College of Medicine, Seoul; ${ }^{3}$ Department of Internal Medicine, Ewha Womans University College \\ of Medicine, Seoul; ‘ Department of Medicine, Samsung Medical Center, Sungkyunkwan University School of Medicine, Seoul; Depart- \\ ments of ${ }^{5}$ Radiology, ${ }^{6}$ Surgery, Seoul National University College of Medicine, Seoul; ${ }^{7}$ Department of Medicine, Chosun University, \\ Gwangju; ${ }^{8}$ Department of Gastroenterology, Asan Medical Center, University of Ulsan College of Medicine, Seoul; ${ }^{9}$ Institute for Digestive \\ Research, Digestive Disease Center, Department of Internal Medicine, Soonchunhyang University College of Medicine, Cheonan; \\ ${ }^{10}$ Cancer Registration and Statistic Branch, National Cancer Control Institute, National Cancer Center, Goyang; ${ }^{11}$ Liver Research Insti- \\ tute, Department of Internal Medicine, Seoul National University College of Medicine, Seoul, Korea
}

Received Feb. 22, 2021

Revised Mar. 3, 2021

Accepted Mar. 4, 2021
Background/Aims: Hepatocellular carcinoma (HCC) is the sixth most common cancer and the second leading cause of cancer-related death in Korea. This study evaluated the characteristics of Korean patients newly diagnosed with HCC in 2015.

Methods: Data from the Korean Primary Liver Cancer Registry (KPLCR), a representative sample of patients newly diagnosed with HCC in Korea, were analyzed. A total of 1,558 patients with HCC registered in the KPLCR in 2015 were investigated.

Results: The median age was 61.0 years (interquartile range, 54.0-70.0 years), and men accounted for $79.7 \%$ of the subjects. Hepatitis B virus infection was the most common underlying liver disease (58.1\%). According to the Barcelona Clinic Liver Cancer (BCLC) staging system, stage $0, A, B, C$, and D HCCs accounted for $14.2 \%, 31.5 \%, 7.6 \%, 39.0 \%$, and $7.8 \%$ of patients, respectively. Transarterial therapy (32.1\%) was the most commonly performed initial treatment, followed by surgical resection (23.2\%), best supportive care $(20.2 \%)$, and local ablation therapy (10.7\%). Overall, $34.5 \%$ of patients were treated in accordance with the BCLC guidelines: $59.2 \%$ in stage $0 / A, 48.4 \%$ in stage $B, 18.1 \%$ in stage $C$, and $71.6 \%$ in stage D. The 1-, 3 -, and 5 -year OS rates were $67.1 \%, 50.9 \%$, and $27.0 \%$, respectively.

Conclusions: In 2015 , approximately $45 \%$ of Korean HCC cases were diagnosed at a very early or early stage, and $35 \%$ of patients underwent potentially curative initial treatment. BCLC guidance was followed in $34.5 \%$ of patients; in patients with stage B or C disease, there was relatively low adherence. (J Liver Cancer 2021;21:58-68)

Keywords: Epidemiology; Hepatocellular carcinoma; Hepatitis B; Korea; Survival

This article was retracted and replaced on June 7, 2022. See supplemental content for versions that show errors and corrections.

\author{
Corresponding author : Jeong-Hoon Lee \\ Department of Internal Medicine, Seoul National University Hospital, 101 Daehak-ro, Jongno-gu, Seoul 03080, Korea \\ Tel. +82-2-2072-2228; Fax. +82-2-743-6701 \\ E-mail: JHLeeMD@snu.ac.kr \\ https://orcid.org/0000-0002-0315-2080 \\ * Jun Sik Yoon and Han Ah Lee contributed equally to this work as co-first authors.
}




\section{INTRODUCTION}

Hepatocellular carcinoma (HCC) is the sixth most common malignancy and the second leading cause of cancer-related deaths in Korea. ${ }^{1-3}$ Cancer-related death from HCC was reported to be the highest among those aged 40-59 years, i.e., those of working age who are economically active. ${ }^{4}$ As such, the annual economic burden of HCC is the highest among all cancers in Korea. ${ }^{5}$ Therefore, it is important to accumulate and provide up-to-date accurate statistics on HCC.

The Korean Primary Liver Cancer Registry (KPLCR) is a random and representative sample of patients who are newly diagnosed with primary liver cancer in Korea. More than 95\% of all cancer cases in Korea are registered in the Korean Central Cancer Registry (KCCR). Among the patients with newly diagnosed primary liver cancer in the KCCR, approximately $15 \%$ were randomly selected after stratification by region and hospital where the diagnosis was made and registered in the KPLCR. Therefore, the KPLCR can be considered to contain a representative group of patients with primary liver cancer in Korea. We previously published reports on HCC-related statistics in Korea using data from the KPLCR between 2008 and $2011^{6}$ and between 2012 and 2014. ${ }^{7}$ In this study, we aimed to investigate the HCC statistics, including baseline characteristics, treatment modalities, and survival, in Korea using data from the KPLCR in 2015.

\section{METHODS}

\section{Patients}

Patients registered in the KPLCR between January 1, 2015, and December 31, 2015, were selected. The details of the KPLCR have been presented in our previous reports. ${ }^{6,7}$ HCC was diagnosed based on histological evidence or using dynamic computed tomography (CT) and/or magnetic resonance imaging (MRI) findings (nodule $>1 \mathrm{~cm}$ with arterial hypervascularity and portal-/delayed-phase washout).

In 2015, a total of 1,558 patients were registered in the KPLCR. Among these patients with HCC, we excluded 1) patients who received their initial treatment more than 120 days from the date of diagnosis ( $\mathrm{n}=38$ ) and 2) patients lacking information on treatment modality $(n=2)$. Finally, 1,518 patients with HCC were included in the treatment group. The treatment group consisted of patients who received active treatment $(n=1,211)$ and those who received best supportive care ( $\mathrm{n}=307)$ (Supplementary Fig. 1). Thirty-eight patients who received their initial treatment more than 120 days after the first HCC diagnosis were initially excluded from the treatment group and subsequent survival analyses because they were likely to receive treatment at a more advanced tumor stage than their initial tumor stage. The Institutional Review Board (IRB) of Seoul National University Hospital decided to waive the need for IRB approval and written informed consent (IRB No. 2204-110-1317) because the KPLCR data were collected anonymously as part of the KCCR in accordance with the Cancer Control Act.

\section{Data collection and definitions}

Patient information was obtained from medical records from each hospital where the diagnosis of HCC was made. Well-trained KCCR-registry data recorders from each hospital investigated the medical records. Data were extracted using a standardized case record form and validated by statisticians affiliated with the KCCR and KPLCR. Data were collected for variables including baseline characteristics such as demographic, laboratory, and tumor variables; and treatment-related factors such as treatment modalities and overall survival (OS) of the patients. All tumor characteristics were assessed using diagnostic imaging, such as dynamic CT or MRI. The modified Union for International Cancer Control (mUICC) staging system and Barcelona Clinic Liver Cancer (BCLC) staging system were adopted as staging systems. OS was measured from the date of HCC diagnosis until death from any cause. Death certificate data were obtained from the national statistical data collected by the Korean Ministry of Government Administration and Home Affairs. Individual patients' data were identified using 13-digit unique resident registration numbers issued to all Koreans. The data cutoff date was December 31, 2020. 
Table 1. Baseline characteristics of the study population $(n=1,558)$

\begin{tabular}{l} 
Variable \\
\hline Demographic variable \\
Age (years) \\
Sex (male) \\
Diabetes \\
Hypertension \\
Body mass index $\left(\mathrm{kg} / \mathrm{m}^{2}\right)$ \\
Smoking \\
Etiology \\
HBV \\
HCV \\
Alcohol \\
Others
\end{tabular}

$\begin{array}{cc}\text { Performance status }^{\dagger} & (\mathrm{n}=1,150, \text { missing }=408) \\ 0 & 848(73.7) \\ 1 & 204(17.7) \\ 2 & 64(5.6) \\ 3 & 21(1.8) \\ 4 & 13(1.1)\end{array}$

Ascites

$\begin{array}{lc}\text { None } & 1,211(77.7) \\ \text { Mild } & 215(13.8) \\ \text { Moderate to severe } & 132(8.5)\end{array}$

Encephalopathy

None

( $n=1,556$, missing $=2)$

Mild to moderate (grade 1 or 2)

$1,518(97.6)$

Severe (grade 3 or 4 )

$28(1.8)$

$10(0.6)$

\begin{tabular}{lc} 
Laboratory variable & \\
Total bilirubin (mg/dL) & $0.9(0.6-1.4)$ \\
Serum albumin (g/dL) & $3.9(3.4-4.3)$ \\
Alanine aminotransferase (IU/L) & $35.0(23.0-57.0)$ \\
Platelet count (10 $/ \mathrm{L})$ & $155.0(107.0-208.5)$ \\
Prothrombin time (INR) & $1.09(1.03-1.19)$ \\
Creatinine (mg/dL) & $0.86(0.73-1.00)$ \\
Sodium (mmol/L) & $139(137-141)$ \\
Glucose (mg/dL) & $100(71-127)$ \\
Total cholesterol (mg/dL) & $154(130-186)$ \\
Child-Turcotte-Pugh class & $(\mathrm{n}=1,499$, missing=59) \\
A & $1,098(73.2)$ \\
B & $332(22.2)$ \\
C & $69(4.6)$ \\
\hline
\end{tabular}

Table 1. continued

\begin{tabular}{|c|c|}
\hline Variable & Total $(n=154)$ \\
\hline MELD score & $8.0(7.0-10.0)$ \\
\hline MELD-Na score & $10.0(8.0-13.0)$ \\
\hline \multicolumn{2}{|l|}{ Tumor variable } \\
\hline Alpha-fetoprotein (ng/mL) & $19.9(4.7-370.3)$ \\
\hline PIVKA-II (mAU/mL) & $102.0(28.0-1,835.5)$ \\
\hline Tumor number & $(n=1,556$, missing $=2)$ \\
\hline 1 & $955(61.4)$ \\
\hline 2 & $201(12.9)$ \\
\hline 3 & $50(3.2)$ \\
\hline 4 & $14(0.9)$ \\
\hline$\geq 5$ & $336(21.6)$ \\
\hline Maximal tumor diameter (cm) & $3.4(2.0-7.0)$ \\
\hline Portal vein invasion & $365(23.4)$ \\
\hline Hepatic vein invasion & $80(5.1)$ \\
\hline Bile duct invasion & $49(3.1)$ \\
\hline Lymph node metastasis & $96(6.2)$ \\
\hline Distant metastasis & $165(10.6)$ \\
\hline Modified UICC Stage & $(\mathrm{n}=1,544$, missing $=14)$ \\
\hline Stage I & $249(16.1)$ \\
\hline Stage II & $592(38.3)$ \\
\hline Stage III & $352(22.8)$ \\
\hline Stage IV-A & $188(12.2)$ \\
\hline Stage IV-B & $163(10.6)$ \\
\hline BCLC stage & $(n=1,242$, missing $=316)$ \\
\hline 0 & $176(14.2)$ \\
\hline A & $391(31.5)$ \\
\hline B & $94(7.6)$ \\
\hline C & $484(39.0)$ \\
\hline D & $97(7.8)$ \\
\hline
\end{tabular}

Values are presented as median (interquartile range) or number (\%). HBV, hepatitis B virus; HCV, hepatitis C virus; INR, international normalized ratio; MELD, Model for End stage Liver Disease; PIVKA-II, protein induced by vitamin K absence-II; UICC, Union for International Cancer Control; BCLC, Barcelona Clinic Liver Cancer.

*Patients co-infected with HBV and HCV $(n=87)$ were also included; ${ }^{\dagger}$ Performance status was classified as follows: 0 , fully active without symptoms; 1 , ambulatory with symptoms; 2 , bedridden $<50 \%$ of the time; 3 , bedridden $>50 \%$ of the time but capable of self-care; 4 , bedridden $100 \%$ of the time and incapable of self-care. 


\section{Statistical analysis}

Data are presented as number (percentage [\%]) or median (interquartile range $[\mathrm{IQR}]$ ), as appropriate. The significance of differences between continuous and categorical variables was examined using Student's $t$-test (or Mann-Whitney $U$ test) and the chi-squared test (or Fisher's exact test), respectively. The OS of patients was evaluated using the KaplanMeier method, and survival differences were analyzed using the log-rank test. All statistical analyses were conducted using SPSS ver. 21.0 (IBM Corp., Armonk, NY, USA) and R version 3.5.2 (http://www.rproject.org). Two-sided $P$-values $<0.05$ were considered to indicate statistical significance.

\section{RESULTS}

\section{Baseline characteristics}

The baseline characteristics of the study population are summarized in Table 1 . The median age was 61 years (IQR, 54-70 years), and the study population was predominantly male $(79.7 \%)$. Hepatitis B virus (HBV) infection was the most common etiology (58.1\%), followed by alcohol abuse (16.8\%), hepatitis C virus (8.2\%), and others (17.0\%). When liver function was classified according to the Child-Turcotte-Pugh (CTP) score, $73.2 \%, 22.2 \%$, and $4.6 \%$ of patients were at CTP classes A, B, and C, respectively. The median AFP level was 19.9 ng/mL (IQR, 4.7-370.3 ng/mL). According to the mUICC staging system, stages I, II, III, IV-A, and IV-B accounted for 16.1\%, 38.3\%, 22.8\%, 12.2\%, and 10.6\% of patients, respectively. According to the BCLC staging system, stage C $(39.0 \%)$ was the most common, followed by stage A $(31.5 \%)$, stage $0(14.2 \%)$, stage D $(7.8 \%)$, and stage B (7.6\%). A single tumor was observed in $61.4 \%$ of the patients and the median maximal diameter of tumors was 3.4 $\mathrm{cm}$ (IQR, 2.0-7.0 cm). On baseline images, portal vein and hepatic vein invasion was seen in $23.4 \%$ and $5.1 \%$ of patients, respectively. Bile duct invasion was noted in $3.1 \%$ of patients. Lymph node metastasis and distant metastasis were seen in $6.2 \%$ and $10.6 \%$ of patients, respectively.

\section{Initial treatment modality}

Table 2 presents the initial treatment modalities in the treatment group. Surgical treatments, including resection $(23.2 \%)$ and liver transplantation $(0.7 \%)$, were performed in approximately one-quarter of patients as the initial treatment. Locoregional treatments, including percutaneous ablative treatment $(10.7 \%)$, external beam radiation therapy (1.4\%), and transarterial treatment (32.1\%), were applied in approximately $45 \%$ of patients. The vast majority of percutaneous local ablation therapies were radiofrequency ablation $(97.5 \%)$. Among the transarterial therapies, $82.8 \%$ were conventional transarterial chemoembolization, $12.7 \%$ were transarterial radioembolization, and 2.9\% were drug-eluting bead transarterial chemoembolization. Systemic treatment

Table 2. Distribution of initial treatment modalities among patients in the treatment group $(n=1,518)$

\begin{tabular}{|c|c|}
\hline Treatment modality & Value \\
\hline Surgical resection & $352(23.2)$ \\
\hline Liver transplantation & $11(0.7)$ \\
\hline Local ablation therapy & $162(10.7)$ \\
\hline Radiofrequency ablation & 158 \\
\hline Percutaneous ethanol injection & 1 \\
\hline Other local ablation & 3 \\
\hline Transarterial therapy & $487(32.1)$ \\
\hline Conventional TACE & 403 \\
\hline TACE with drug-eluting beads & 14 \\
\hline Radioembolization & 62 \\
\hline Hepatic arterial infusion chemotherapy & 8 \\
\hline Combination therapy* & $20(1.3)$ \\
\hline Systemic therapy & $103(6.8)$ \\
\hline Sorafenib & 100 \\
\hline Other systemic agents & 3 \\
\hline External beam radiation therapy & $22(1.4)$ \\
\hline Best supportive care & $307(20.2)$ \\
\hline Miscellaneous therapies $^{\dagger}$ & $54(3.6)$ \\
\hline \multicolumn{2}{|c|}{$\begin{array}{l}\text { Values are presented as number (\%) unless otherwise indicated. } \\
\text { TACE, transarterial chemoembolization. } \\
{ }^{*} \text { Combination therapy was defined as a combined treatment with } \\
\text { local ablation therapy and transarterial therapy; }{ }^{\dagger} \text { Miscellaneous } \\
\text { therapies were defined as unclassifiable treatment modalities (i.e., } \\
\text { combination therapies other than transarterial therapy and local } \\
\text { ablation therapy). }\end{array}$} \\
\hline
\end{tabular}


was performed in $6.8 \%$ of patients, and $20.2 \%$ underwent best supportive care only.

The treatment modalities according to BCLC stage are shown in Fig. 1. For BCLC stage 0, 61.4\% of patients underwent potentially curative treatment (treatment recommended by the BCLC guidelines), including surgical resection (26.5\%) or local ablation therapy (34.9\%), but $31.9 \%$ of cases were treated with transarterial treatment. For BCLC stage A, $58.2 \%$ of patients were treated with surgical resection

Total

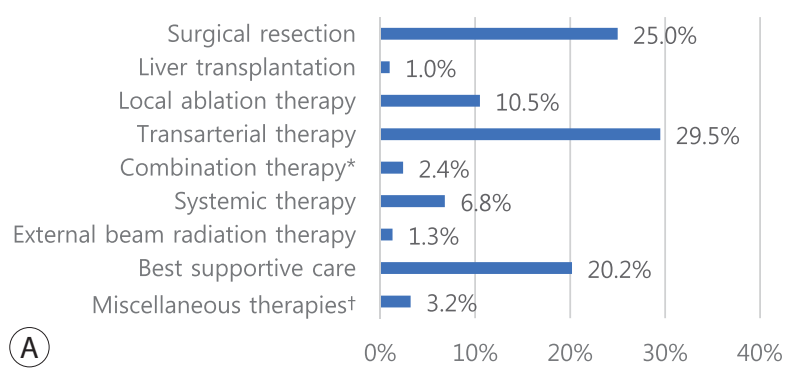

BCLC stage $A$

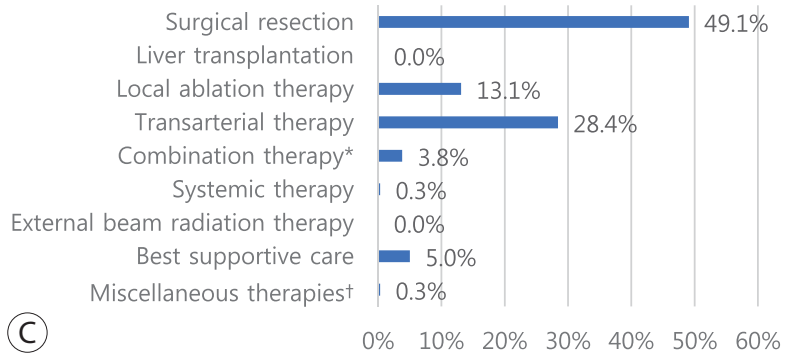

BCLC stage $C$

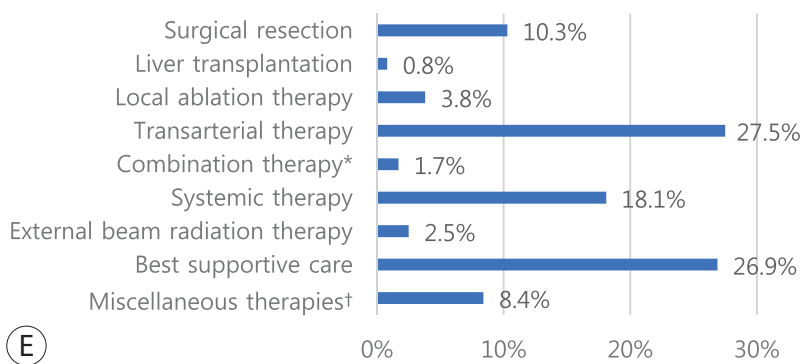

(47.0\%) and local ablation therapy (11.2\%), which are the recommended treatments. For BCLC stage B, $48.4 \%$ of patients were treated with transarterial treatment (recommended treatment), but $23.1 \%$ were treated with surgical resection. For BCLC stage C, only $18.1 \%$ of patients underwent systemic treatment (recommended treatment), and 30.0\% were treated with transarterial treatments the initial treatment. Among BCLC stage D patients, $71.6 \%$ received best supportive care. The overall rate of adherence to the BCLC-

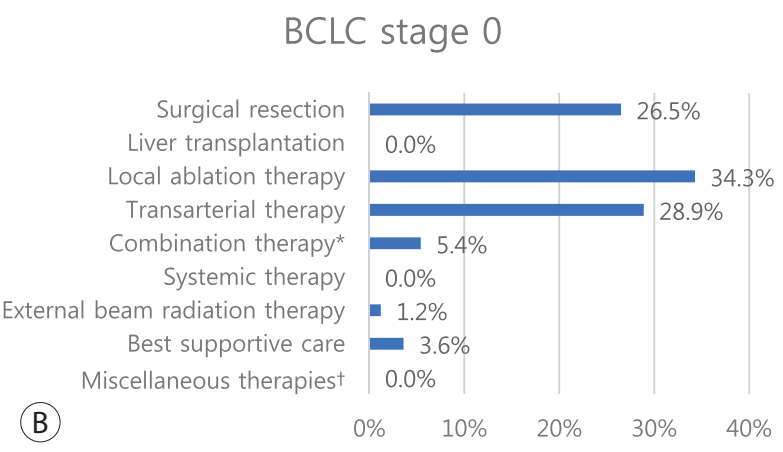

BCLC stage $B$

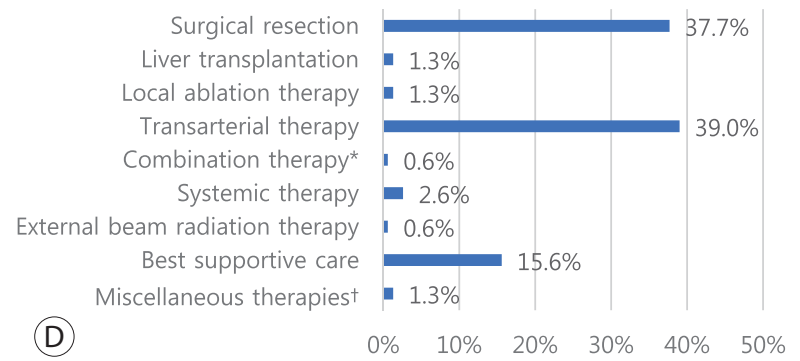

BCLC stage $D$

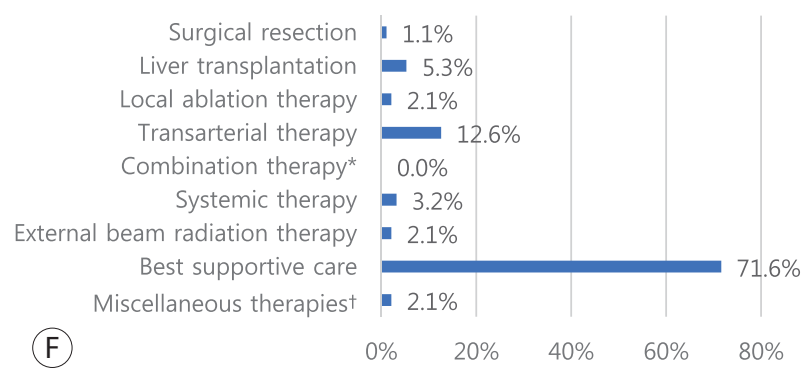

Figure 1. Initial treatment modality of the study population (A) and subgroups according to the Barcelona Clinic Liver Cancer (BCLC) stage 0 (B), stage $A(C)$, stage $B(D)$, stage $C(E)$, and stage $D(F)$. ${ }^{*}$ Combination therapy was defined as a combined treatment with local ablation therapy and transarterial therapy; ${ }^{\dagger}$ Miscellaneous therapies were defined as unclassifiable treatment modalities (i.e., combination therapies other than transarterial therapy and local ablation therapy). 
Table 3. Overall survival rates (\%) in the study population according to baseline characteristics and initial treatment modalities

\begin{tabular}{|c|c|c|c|c|c|c|c|}
\hline \multirow{2}{*}{ Variable } & \multirow{2}{*}{$\mathrm{n}(\%)$} & \multirow{2}{*}{$\begin{array}{c}\text { Median OS } \\
(95 \% \mathrm{Cl} \text {, months) }\end{array}$} & \multicolumn{5}{|c|}{ Year } \\
\hline & & & 1 & 2 & 3 & 4 & 5 \\
\hline All patients & $1,558(100)$ & $3.15(2.62-3.65)$ & 67.1 & 57.1 & 50.9 & 45.9 & 27.0 \\
\hline \multicolumn{8}{|c|}{ Age (years; $n=1,558$, missing values $=0$ ) } \\
\hline$<40$ & $34(2.2)$ & NR (1.59-NR) & 73.5 & 61.8 & 58.8 & 58.8 & 55.6 \\
\hline $40-49$ & $178(11.4)$ & $2.75(1.63-4.54)$ & 60.1 & 53.9 & 48.9 & 45.5 & 40.6 \\
\hline $50-59$ & $473(30.4)$ & $4.45(4.02-4.90)$ & 68.9 & 62.2 & 58.1 & 54.5 & 37.4 \\
\hline $60-69$ & $440(28.2)$ & $4.06(3.04-4.35)$ & 71.6 & 62.7 & 55.0 & 50.4 & 28.0 \\
\hline$\geq 70$ & $443(27.8)$ & $1.76(1.49-2.14)$ & 62.8 & 46.7 & 39.0 & 30.9 & 11.9 \\
\hline \multicolumn{8}{|c|}{ Sex $(n=1,558$, missing values $=0)$} \\
\hline Male & $1,241(79.7)$ & $3.01(2.42-3.50)$ & 66.2 & 55.8 & 50.0 & 44.8 & 24.6 \\
\hline Female & $317(20.3)$ & $3.95(2.71-4.68)$ & 70.3 & 61.8 & 54.3 & 50.1 & 36.9 \\
\hline \multicolumn{8}{|c|}{ Etiology $(n=1,558$, missing values $=0$ ) } \\
\hline$H B V^{*}$ & $905(58.1)$ & $4.26(3.52-4.69)$ & 68.7 & 60.1 & 55.6 & 51.8 & 33.5 \\
\hline $\mathrm{HCV}$ & $127(8.2)$ & $2.02(1.60-2.76)$ & 67.7 & 51.2 & 39.4 & 36.2 & 21.2 \\
\hline Alcohol & $261(16.8)$ & $1.91(1.45-2.54)$ & 60.9 & 48.7 & 41.4 & 33.7 & 19.2 \\
\hline Others & $265(17.0)$ & $2.97(2.20-3.77)$ & 67.3 & 57.9 & 49.6 & 42.5 & 22.1 \\
\hline \multicolumn{8}{|c|}{ Child-Turcotte-Pugh classification ( $n=1,499$, missing values=59) } \\
\hline A & $1,098(73.3)$ & $4.64(4.35-4.81)$ & 79.5 & 69.5 & 63.1 & 57.6 & 35.7 \\
\hline B & $332(22.1)$ & $0.50(0.38-0.70)$ & 39.2 & 28.0 & 21.7 & 18.1 & 0.0 \\
\hline C & $69(4.6)$ & $0.13(0.10-0.22)$ & 14.5 & 13.0 & 8.7 & 5.8 & 2.9 \\
\hline \multicolumn{8}{|c|}{ Alpha-fetoprotein ( $\mathrm{ng} / \mathrm{mL} ; \mathrm{n}=1,459$, missing values=99) } \\
\hline$<11.45$ & $581(39.9)$ & $4.75(4.44-\mathrm{NR})$ & 83.3 & 74.5 & 68.3 & 61.1 & 40.9 \\
\hline $11.45-19.99$ & $107(7.3)$ & $4.70(3.35-N R)$ & 72.0 & 65.4 & 60.7 & 56.1 & 35.9 \\
\hline $20.00-99.99$ & $229(15.7)$ & $4.00(2.79-4.76)$ & 77.3 & 65.5 & 56.3 & 50.2 & 26.5 \\
\hline 100.00-199.99 & $71(4.9)$ & $2.00(1.40-3.59)$ & 63.4 & 50.7 & 42.3 & 36.6 & 29.1 \\
\hline 200.00-399.99 & $78(5.3)$ & $3.81(1.76-N R)$ & 77.3 & 57.7 & 53.8 & 48.7 & 30.2 \\
\hline$\geq 400.00$ & $393(26.9)$ & $0.48(0.41-0.63)$ & 38.2 & 28.0 & 24.4 & 22.9 & 7.6 \\
\hline \multicolumn{8}{|c|}{ Modified UICC stage $(n=1,544$, missing values $=14)$} \\
\hline Stage I & $249(16.1)$ & 4.98 (4.89-NR) & 93.1 & 87.5 & 80.6 & 73.4 & 42.3 \\
\hline Stage II & $592(38.3)$ & 4.90 (4.75-NR) & 88.8 & 80.2 & 73.9 & 67.2 & 40.5 \\
\hline Stage III & $352(22.8)$ & $1.67(1.40-2.02)$ & 61.8 & 45.3 & 38.0 & 33.6 & 19.9 \\
\hline Stage IV-A & $188(12.2)$ & $0.32(0.27-0.38)$ & 21.8 & 12.2 & 6.9 & 4.8 & 0.4 \\
\hline Stage IV-B & $163(10.6)$ & $0.25(0.18-0.30)$ & 16.0 & 8.0 & 4.3 & 3.9 & 0.2 \\
\hline \multicolumn{8}{|c|}{$B C L C$ stage $(n=1,242$, missing $=316)$} \\
\hline 0 & $176(14.2)$ & 4.98 (4.89-NR) & 94.3 & 88.6 & 81.8 & 75.0 & 42.9 \\
\hline A & $391(31.5)$ & 4.88 (4.76-NR) & 92.6 & 84.9 & 79.5 & 74.2 & 35.8 \\
\hline B & $94(7.6)$ & $2.61(1.65-4.43)$ & 77.5 & 55.3 & 45.7 & 40.4 & 29.4 \\
\hline C & $484(39.0)$ & $0.64(0.48-0.87)$ & 42.4 & 31.4 & 24.6 & 21.1 & 13.7 \\
\hline D & $97(7.8)$ & $0.14(0.12-0.29)$ & 18.6 & 15.5 & 10.3 & 7.2 & 0.0 \\
\hline \multicolumn{8}{|c|}{ Initial treatment modalities $(n=1,518$, missing values $=40$ ) } \\
\hline Surgical resection & $352(23.2)$ & NR (4.99-NR) & 94.3 & 89.2 & 86.1 & 83.0 & 54.6 \\
\hline
\end{tabular}


Table 3. Continued

\begin{tabular}{|c|c|c|c|c|c|c|c|}
\hline \multirow{2}{*}{ Variable } & \multirow{2}{*}{ n (\%) } & \multirow{2}{*}{$\begin{array}{c}\text { Median OS } \\
\text { (95\% Cl, months) }\end{array}$} & \multicolumn{5}{|c|}{ Year } \\
\hline & & & 1 & 2 & 3 & 4 & 5 \\
\hline Liver transplantation & $11(0.7)$ & NR (2.79-NR) & 81.8 & 81.8 & 63.6 & 63.6 & 63.6 \\
\hline Local ablation therapy & $162(10.7)$ & NR (4.76-NR) & 92.6 & 89.5 & 83.3 & 74.1 & 53.0 \\
\hline Transarterial therapy & $487(32.1)$ & $2.76(2.37-3.46)$ & 77.0 & 60.2 & 49.1 & 41.7 & 16.1 \\
\hline Combination therapy $^{\dagger}$ & $20(1.3)$ & 4.90 (4.76-NR) & 95.0 & 80.0 & 80.0 & 70.0 & 37.3 \\
\hline Systemic therapy & $103(6.8)$ & $0.32(0.27-0.39)$ & 14.6 & 4.9 & 2.9 & 2.0 & 2.0 \\
\hline External beam radiation therapy & $22(1.4)$ & $0.38(0.29-1.83)$ & 31.8 & 22.7 & 9.1 & 9.1 & 4.6 \\
\hline Best supportive care & $307(20.2)$ & $0.20(0.17-0.28)$ & 21.8 & 14.7 & 12.7 & 8.8 & 5.1 \\
\hline Miscellaneous therapies ${ }^{\ddagger}$ & $54(3.6)$ & 2.40 (1.52-NR) & 68.5 & 53.7 & 44.4 & 44.4 & 38.1 \\
\hline
\end{tabular}

OS, overall survival; Cl, confidence interval; NR, not reached; HBV, hepatitis B virus; HCV, hepatitis C virus; UICC, Union for International Cancer Control; BCLC, Barcelona Clinic Liver Cancer.

*Patients co-infected with HBV and HCV $(n=13)$ were also included; ${ }^{\dagger}$ Combination therapy was defined as a combined treatment with local ablation therapy and transarterial therapy; ${ }^{\ddagger}$ Miscellaneous therapies were defined as unclassifiable treatment modalities (i.e., combination therapies other than transarterial therapy and local ablation therapy).

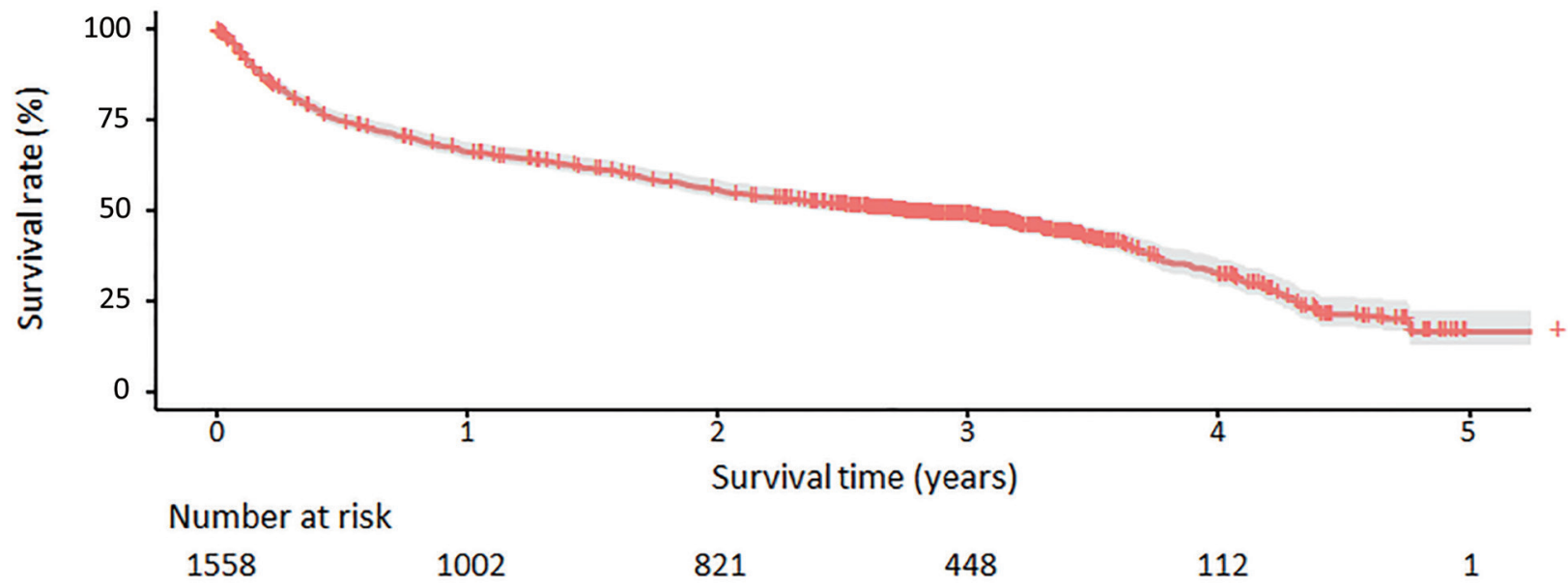

Figure 2. Overall survival curves for Korean patients with hepatocellular carcinoma.

suggested treatment was $34.5 \%$. The rates of adherence to the BCLC treatment guidelines according to each BCLC stage are shown in Supplementary Table 1. Adherence to the BCLC guidelines was relatively high for BCLC stages 0/A (59.2\%) and D (71.6\%). However, for BCLC stage C, the rate of adherence to the recommended treatment (i.e., systemic treatment) was only $18.1 \%$.

\section{Overall survival}

Table 3 presents the median OS and annual OS rates for the entire study population and the OS rates according to baseline characteristics. The median OS was 37.8 months (95\% CI, 31.4-43.8 months), and the 1-, 3-, and 5-year OS rates were $67.1 \%, 50.9 \%$, and $27.0 \%$, respectively (Fig. 2). All survival curves showed significant survival differences according to CTP class (Fig. 3A), mUICC stage (Fig. 3B), BLCL stage after combining stages 0 and A (Fig. 3C), and AFP levels (Fig. 3D) (all log-rank test, $P<0.001$ ). The survival curves of BCLC stages 0 and A did not show any significant survival differences (log-rank test, $P=0.37$ ). We also performed post hoc analyses of log-rank tests for multiple comparisons between each survival curve and found significant survival differences between all CTP classes, between all 

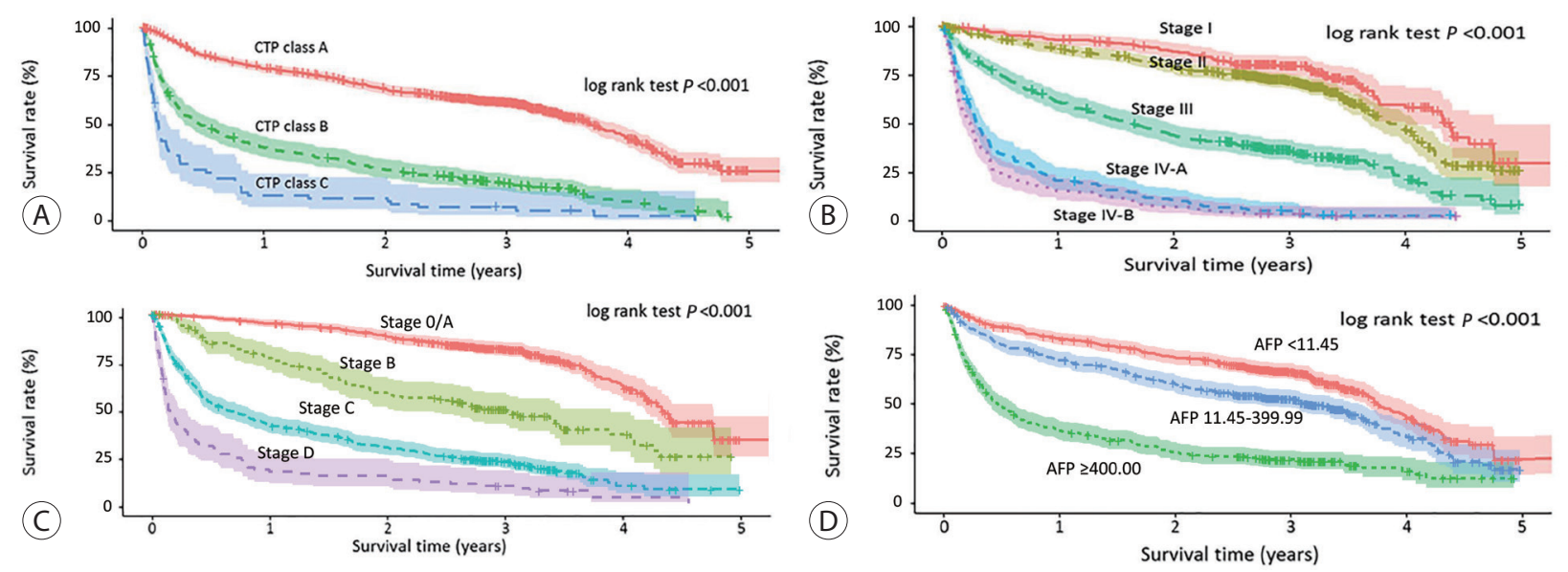

Figure 3. Overall survival curves for the subgroups divided by the Child-Turcotte-Pugh (CTP) classes (A), modified Union for International Cancer Control stage (B), Barcelona Clinic Liver Cancer stage (C), and serum levels of alpha-fetoprotein (AFP) (D).

Table 4. Distribution of initial treatment modalities among patients in the treatment group $(n=1,518)$

\begin{tabular}{|c|c|c|c|}
\hline Variable & n (\%) & Median OS (95\% Cl, months) & Interquartile range \\
\hline \multicolumn{4}{|l|}{ BCLC stage $0 / A$} \\
\hline Local ablation therapy & $101(18.4)$ & NR (57.1-NR) & 49.2-NR \\
\hline Surgical resection & $224(40.8)$ & 59.9 (59.9-NR) & 57.7-NR \\
\hline Local ablation therapy+Surgical resection & $325(59.2)$ & 59.9 (59.9-NR) & 56.2-NR \\
\hline \multicolumn{4}{|l|}{ BCLC stage $B$} \\
\hline Transarterial therapy & $44(48.4)$ & $27.0(19.2-53.2)$ & $13.6-58.2$ \\
\hline \multicolumn{4}{|l|}{ BCLC stage $C$} \\
\hline Systemic therapy & $86(18.1)$ & $4.1(3.2-5.0)$ & $1.9-7.3$ \\
\hline \multicolumn{4}{|l|}{ BCLC stage D } \\
\hline Best supportive care & $68(71.6)$ & $1.3(1.0-1.7)$ & $0.6-3.1$ \\
\hline
\end{tabular}

OS, overall survival; Cl, confidence interval; BCLC, Barcelona Clinic Liver Cancer; NR, not reached.

HCC stages, and between all AFP levels (all pairwise log-rank comparisons, $P<0.001)$. When we analyzed the median survival of patients who received initial treatment as recommended by the BCLC guidelines (Table 4), it was 59.9 months (IQR, 56.2 months-not reached) in BCLC stages 0/ A, 27.0 months (IQR, 13.6-58.2 months) in stage B, 4.1 months (IQR, 1.9-7.3 months) in stage $\mathrm{C}$, and 1.3 months (IQR, 0.6-3.1 months) in stage D.

\section{DISCUSSION}

The present study investigated HCC-related statistics, including baseline characteristics, treatment modalities, and survival, in Korea in 2015. We analyzed 1,558 patients with HCC registered in the KPLCR in 2015, who are representative of the general population with HCC in Korea. We showed that $45.7 \%$ of patients with HCC were diagnosed at a very early or early stage (BCLC stage 0 or A) and could be candidates for curative treatment; however, a similar proportion of patients (39\%) was diagnosed at an advanced stage (BCLC stage C). Transarterial therapy (32.1\%) was the most commonly performed initial treatment, followed by surgical resection (23.2\%), best supportive care (20.2\%), and local ablation therapy (10.7\%). The 1-, 3-, and 5-year OS rates were $67.1 \%, 50.9 \%$, and $27.0 \%$, respectively. BCLC guidance for treatment was followed in $34.5 \%$ of patients. The treat- 
ment of patients with BCLC stage B and C showed relatively low adherence to this guidance.

The trends in HCC-related statistics in Korea have changed over time. We previously published reports on HCC-related statistics in Korea using data from the KPLCR between 2008 and 2011 and between 2012 and 2014. ${ }^{6.7}$ The HCC-related statistics using the data in the KPLCR between 2003 and 2005 are also available from a website established by the Korean Liver Cancer Study Group (http://www.livercancer.or.kr). Therefore, we were able to compare the HCC statistics for 2003-2005, 2008-2011, and 2012-2014 with those in 2015. We found that several characteristics, including the etiology of HCC, underlying liver function, treatment modalities, and rates of adherence to BCLC treatment guidelines, changed significantly over time. The prevalence of HBV-related HCC gradually decreased over time, while the prevalence of HCC caused by etiologies other than viral hepatitis or alcohol abuse (e.g., non-alcoholic fatty liver disease [NAFLD]) increased over time. The rates of HBV-related HCC for the periods 2003-2005, 2008-2011, 2012-2014, and 2015 were $72.3 \%, 72.0 \%, 59.1 \%$, and $58.1 \%$, respectively. In Korea, after the introduction of HBV vaccination in 1983 and nationwide immunization in 1995, the rate of positivity for hepatitis B surface antigen dropped from $8-10 \%$ in the 1980 s to $3 \%$ in $2016 .{ }^{8-10}$ However, the prevalence of NAFLD is approximately $16-33 \%$ and is steadily increasing in Korea. ${ }^{11,12}$ Therefore, the prevalence of HCC caused by NAFLD is expected to increase in Korea. The rate of HCC caused by etiologies other than viral hepatitis or alcohol abuse increased from $5.7 \%$ in 2003-2005 to $17.0 \%$ in 2015.

The proportion of patients with preserved liver function (CTP A) increased over time. The proportion of patients with CTP A for 2003-2005, 2008-2011, 2012-2014, and 2015 were $64.2 \%, 71.0 \%, 72.9 \%$, and $73.2 \%$, respectively. This can be explained by advances in antiviral treatment and management of liver cirrhosis in Korea. Entecavir and tenofovir, two of the most potent antiviral agents against HBV, were introduced in 2007 and 2012, respectively. ${ }^{13}$ The application of potent antiviral agents can successfully inhibit viral replication, thereby reducing liver inflammation and fibrosis.

As the underlying liver function improved in patients with
HCC over time, the proportion of patients receiving potentially curative treatment as initial treatment also increased. The proportions of patients with HCC receiving curative treatment (i.e., surgical resection, liver transplantation, and percutaneous ablative treatment) for 2003-2005, 2008-2011, 2012-2014, and 2015 were $17.1 \%, 28.2 \%, 31.5 \%$, and 34.6\%, respectively. The rate of patients receiving systemic treatment also increased from $0.8 \%$ in $2003-2005$ to $6.8 \%$ in 2015 . Considering that approximately $40 \%$ of HCC cases are identified at an advanced stage and that novel systemic agents for first-line treatment (e.g., lenvatinib and atezolizumab plus bevacizumab) and second-line or later treatment (e.g., regorafenib, nivolumab, ramucirumab, pembrolizumab, and cabozantinib) have recently been introduced, ${ }^{14-20}$ systemic treatment is expected to become more widely used for patients with HCC in the near future. In contrast, although transarterial treatment was the most commonly used treatment modality in all periods, the proportion of patients with HCC receiving transarterial treatment as the initial treatment has been gradually decreasing over time $(49.5 \%, 41.7 \%$, $37.5 \%$, and $32.1 \%$ for the periods 2003-2005, 2008-2011, 2012-2014, and 2015, respectively).

The overall adherence rates for the periods 2008-2011, 2012-2014, and 2015 were $37.7 \%, 38.2 \%$, and 34.5\%, respectively. As the proportion of patients with HCC receiving curative treatment has increased over time, the adherence rate of patients at BCLC stage 0/A also increased (47.4\%, 54.8\%, and 59.2\% for the periods 2008-2011, 2012-2014, and 2015, respectively). The adherence rate of patients at BCLC stage $\mathrm{C}$ has also increased as the number of HCC patients receiving systemic treatment has increased $(9.5 \%, 11.7 \%$, and $18.1 \%$ for the periods 2008-2011, 2012-2014, and 2015, respectively). However, unexpectedly, the adherence rate of patients at BCLC stage B decreased over time $(62.8 \%, 55.1 \%$, and $48.4 \%$ for the periods 2008-2011, 2012-2014, and 2015, respectively). This trend might be due to an increase in the proportion of patients with HCC at BCLC stage B who underwent surgical resection $(10.2 \%, 21.1 \%$, and $23.1 \%$ for the periods 2008-2011, 2012-2014, and 2015, respectively). A previous meta-analysis indicated that surgical resection provides a significant survival benefit over transarterial treat- 
ment in patients with BCLC stage B HCC when the HCC is resectable. ${ }^{21}$ Because HCCs at BCLC stage B are highly heterogeneous, more stratified and individualized treatment guidelines are warranted, considering liver function, tumor extent, and the level of vascular invasion.

In conclusion, we have shown that in 2015, approximately $45 \%$ of HCCs were diagnosed at a very early or early stage, and $35 \%$ of patients received potentially curative treatment as their initial treatment. The BCLC guidelines for treatment were followed in $34.5 \%$ of patients with HCC; there was relatively low adherence to these guidelines in patients with BCLC stage B or C HCC. We believe that the results and highlighted trends in the characteristics of HCC in Korea will contribute to improve the management of this disease and promote further study.

\section{AUTHORSHIP STATEMENT}

\section{1) Jeong-Hoon Lee takes responsibility for the integri- ty of the work as a whole, from inception to pub- lished article.}

\section{2) Specific author contributions}

Conception: Jun Sik Yoon, Han Ah Lee, and Jeong-Hoon Lee; study design: Jun Sik Yoon, Han Ah Lee, and JeongHoon Lee; participation in patient management and data collection: Jun Sik Yoon, Han Ah Lee, Hwi Young Kim, Dong Hyun Sinn, Dong Ho Lee, Ju-Yeon Cho, Jonggi Choi, Young Chang, Suk Kyun Hong, Hyun-Joo Kong, Young-Joo Won, Eunyang Kim, and Jeong-Hoon Lee; contribution to the data acquisition, responsibility for writing the paper, and statistical analysis: Jun Sik Yoon, Han Ah Lee, and JeongHoon Lee

\section{3) All authors have reviewed the paper and approved the final version.}

\section{ACKNOWLEDGMENT}

The database used in this study was provided by the Korean Central Cancer Registry, Ministry of Health and Welfare,
South Korea, and the Korean Liver Cancer Association.

\section{Conflicts of Interest}

The authors have no financial conflicts of interest.

\section{REFERENCES}

1. Kweon SS. Updates on Cancer Epidemiology in Korea, 2018. Chonnam Med J 2018;54:90-100.

2. Statistics Korea. Annual report on the causes of death statistics 2019 [Internet]. Daejeon (KR): Statistics Korea; [cited 2021 Feb 1]. Available from: https://kosis.kr.

3. National Cancer Center. Korea central cancer registry. Annual report of cancer statistics in Korea in 2018. [Internet]. Goyang (KR): National Cancer Center; [cited 2021 Feb 1]. Available from: https:// ncc.re.kr/cancerStatsView.ncc.

4. Korean Liver Cancer Association; National Cancer Center. 2018 Korean Liver Cancer Association-National Cancer Center Korea Practice Guidelines for the Management of Hepatocellular Carcinoma. Gut Liver 2019;13:227-299.

5. Lee KS, Chang HS, Lee SM, Park EC. Economic burden of cancer in Korea during 2000-2010. Cancer Res Treat 2015;47:387-398.

6. Yoon JS, Lee HA, Park JY, Kim BH, Lee IJ, Chon YE, et al. Hepatocellular carcinoma in Korea between 2008 and 2011: an analysis of Korean Nationwide Cancer Registry. J Liver Cancer 2020;20:4152.

7. Chon YE, Lee HA, Yoon JS, Park JY, Kim BH, Lee IJ, et al. Hepatocellular carcinoma in Korea between 2012 and 2014: an analysis of data from the Korean Nationwide Cancer Registry. J Liver Cancer 2020;20:135-147.

8. Chun BY, Lee MK, Rho YK. The prevalence of hepatitis B surface antigen among Korean byliterature review. Epidemiol Health 1992;14:70-78.

9. Cho EJ, Kim SE, Suk KT, An J, Jeong SW, Chung WJ, et al. Current status and strategies for hepatitis B control in Korea. Clin Mol Hepatol 2017;23:205-211.

10. Yim SY, Kim JH. The epidemiology of hepatitis B virus infection in Korea. Korean J Intern Med 2019;34:945-953.

11. Korean Association for the Study of the Liver (KASL). KASL clinical practice guidelines: management of nonalcoholic fatty liver disease. Clin Mol Hepatol 2013;19:325-348.

12. Kwak MS, Kim D. Non-alcoholic fatty liver disease and lifestyle modifications, focusing on physical activity. Korean J Intern Med 2018:33:64-74.

13. Korean Association for the Study of the Liver (KASL). KASL clinical practice guidelines for management of chronic hepatitis B. Clin Mol Hepatol 2019;25:93-159.

14. Kudo M, Finn RS, Qin S, Han KH, Ikeda K, Piscaglia F, et al. Len- 
vatinib versus sorafenib in first-line treatment of patients with unresectable hepatocellular carcinoma: a randomised phase 3 noninferiority trial. Lancet 2018;391:1163-1173.

15. Finn RS, Qin S, Ikeda M, Galle PR, Ducreux M, Kim TY, et al. Atezolizumab plus bevacizumab in unresectable hepatocellular carcinoma. N Engl J Med 2020;382:1894-1905.

16. Bruix J, Qin S, Merle P, Granito A, Huang YH, Bodoky G, et al. Regorafenib for patients with hepatocellular carcinoma who progressed on sorafenib treatment (RESORCE): a randomised, doubleblind, placebo-controlled, phase 3 trial. Lancet 2017;389:56-66.

17. El-Khoueiry AB, Sangro B, Yau T, Crocenzi TS, Kudo M, Hsu C, et al. Nivolumab in patients with advanced hepatocellular carcinoma (CheckMate 040): an open-label, non-comparative, phase 1/2 dose escalation and expansion trial. Lancet 2017;389:2492-2502.

18. Zhu AX, Kang YK, Yen CJ, Finn RS, Galle PR, Llovet JM, et al.
Ramucirumab after sorafenib in patients with advanced hepatocellular carcinoma and increased $\alpha$-fetoprotein concentrations (REACH-2): a randomised, double-blind, placebo-controlled, phase 3 trial. Lancet Oncol 2019;20:282-296.

19. Finn RS, Ryoo BY, Merle P, Kudo M, Bouattour M, Lim HY, et al. Pembrolizumab as second-line therapy in patients with advanced hepatocellular carcinoma in KEYNOTE-240: a randomized, doubleblind, phase III trial. J Clin Oncol 2020;38:193-202.

20. Abou-Alfa GK, Meyer T, Cheng AL, El-Khoueiry AB, Rimassa L, Ryoo BY, et al. Cabozantinib in patients with advanced and progressing hepatocellular carcinoma. N Engl J Med 2018;379:54-63.

21. Hyun MH, Lee YS, Kim JH, Lee CU, Jung YK, Seo YS, et al. Hepatic resection compared to chemoembolization in intermediate- to advanced-stage hepatocellular carcinoma: a meta-analysis of highquality studies. Hepatology 2018;68:977-993. 